\title{
Hypoallergenic allergen derivatives of Pru p 3 for immunotherapy of IgE-mediated peach allergy
}

\author{
B Linhart ${ }^{1 *}$, A Gstoettner ${ }^{1}$, C Gamez ${ }^{2}$, I Swoboda ${ }^{1}$, A Mari ${ }^{3}$, N Papadopoulos ${ }^{4}$, R Valenta ${ }^{1}$ \\ From Food Allergy and Anaphylaxis Meeting (FAAM 2013) \\ Nice, France. 7-9 February 2013
}

\section{Background}

IgE-mediated allergy to peach represents a frequent cause of severe anaphylactic reactions in Mediterranean countries. Pru p 3, a nonspecific lipid transfer protein (LTP) from peach, was identified as an important elicitor of IgEmediated food hypersensitivity reactions to peach. Specific immunotherapy is currently the only allergen-specific and disease-modifying treatment of allergies, but has so far not been applied to food allergic patients due to the high risk of IgE-mediated side effects. Hypoallergenic Pru p 3 derivatives have therefore been constructed in order to minimize side-effects, but these molecules were not immunogenic in animal models.

\section{Methods}

Two recombinant Pru p 3-derivatives were developed based on the Pru p 3 amino acid sequence, by introduction of point mutations, or rearrangements of Pru p 3 fragments and oligomerization. Genes encoding the resulting Pru p 3 derivatives were cloned into the expression vector pET27b and expressed in Escherichia coli. The proteins were purified by affinity chromatography and analyzed by SDSPAGE and mass spectrometry. IgE-reactivity was tested by dot blot analysis and immunogenicity was investigated by immunization of rabbits.

\section{Results}

Expression of the two recombinant Pru p 3 derivatives in Escherichia coli yielded high amounts of recombinant proteins. They were purified to homogeneity and exhibited a strongly reduced IgE-reactivity when exposed to sera of peach allergic patients. Upon immunization of rabbits the Pru p 3 derivatives induced a robust IgG antibody response specific for natural Pru p 3.

\section{Conclusion}

The reported recombinant hypoallergenic Pru p 3 derivatives are candidates for specific immunotherapy of peach allergic patients.

\section{Disclosure of interest}

B Linhart: None declared, A Gstoettner: None declared, C Gamez: None declared, I Swoboda: None declared, A Mari: None declared, N Papadopoulos: None declared, R Valenta: Grant/research support from Biomay AG, Vienna, Austria.

\section{Acknowledgement \\ This work was supported by a research grant from Biomay AG, Vienna, Austria.}

\section{Author details}

${ }^{1}$ Dept. of Pathophysiology and Allergy Research, Medical University of Vienna, Vienna, Austria. ${ }^{2}$ Institute for Medical Research, Foundation Jimenez Diaz, Madrid, Spain. ${ }^{3}$ Center for Molecular Allergology, IDI-IRCCS, Rome, Italy. ${ }^{4}$ Allergy Research Center, 2nd Pediatric Department, National \& Kapodistrian University of Athens, Athens, Greece.

Published: 25 July 2013

\section{doi:10.1186/2045-7022-3-S3-P177}

Cite this article as: Linhart et al:: Hypoallergenic allergen derivatives of Pru p 3 for immunotherapy of IgE-mediated peach allergy. Clinical and Translational Allergy 2013 3(Suppl 3):P177. 